Août 1996

\title{
LINEAR EXCHANGE ECONOMIES WITH A CONTINUUM OF AGENTS
}

\author{
Monique FLORENZANO * \\ Emma MORENO GARCÍA **
}

$\mathrm{N}^{0} 9609$

* CNRS-CEPREMAP, 140 rue du Chevaleret, 75013 Paris, France.

** Departamento de Economía, Universidad Carlos III de Madrid, Calle Madrid 126, 28903 Getafe (Madrid), Espagne.

This work was done during reciprocal visits of the authors. Both institutions, CEPREMAP and Universidad Carlos III de Madrid, are gratefully acknowledged for their hospitality. We have benefitted from dicussions with J.M. Bonnisseau and Pascal Gourdel in Paris and C. Nuñez in Madrid. 


\title{
ECONOMIES D'ECHANGE LINEAIRES AVEC UN CONTINU D'AGENTS
}

\section{Résumé}

Le but de ce papier est d'étudier comment varient les prix d'équilibre en fonction des ressources initiales des agents, dans une économie d'échange linéaire dont l'ensemble des agents est un espace mesuré sans atome. Après avoir défini le modèle, on donne des conditions suffisantes d'une force croissante pour l'existence, l'unicité et la continuité des prix d'équilibre. On montre ensuite que, si on se restreint aux économies dont les ressources initiales sont essentiellement bornées et qui n'ont, du point de vue des préférences, qu'un nombre fini de types d'agents, le vecteur des prix d'équilibre est une fonction indéfiniment différentiable des dotations initiales, sur un sous-ensemble ouvert et dense de l'espace de ces dotations. La clef de ce résultat est une formule explicite permettant de calculer le vecteur des prix d'équilibre au voisinage d'une dotation initiale dite "régulière" où ces prix sont connus.

Mots clés : Utilités linéaires - Fonction d'utilié indirecte - Equilibre de Walras - Espace d'agents mesuré sans atome - Théorème de l'application ouverte.

\section{LINEAR EXCHANGE ECONOMIES WITH A CONTINUUM OF AGENTS}

\begin{abstract}
The purpose of this paper is to study how the equilibrium prices vary with respect to the initial endowments in a linear exchange economy with a continuum of agents. We first state the model and give conditions of an increasing strength for existence, uniqueness and continuity of equilibrium prices.Then, if we restrict ourselves to economies with essentially bounded initial endowments and if we assume that there is, from the point of view of preferences, only a finite number of types of agents, we show that, on an open dense subset of the space of initial endowments, the equilibrium price vector is an infinitely differentiable function of the initial endowments. The proof of this claim is based on an explicit formula allowing to compute the equilibrium price vector around a so-called "regular" endowment where it is known.

Key words : Linear utilities - Indirect utility function - Walrasian equilibrium - Atomless measure space of agents - Open mapping theorem.

JEL : C62, D51.
\end{abstract}




\section{Introduction}

Linear exchange economies with a finite number of consumers have been extensively studied (see, for example, Gale (1957, 1960, 1976), Eaves (1976), Cheng (1979) and Cornet (1989)). These economies are interesting in themselves for different models but they are also interesting as a local approximation of an economy with standard differentiable strictly quasi-concave utility functions (see Champsaur-Cornet (1990)). Recently, Bonnisseau and Jofré (1994) have proved that, on an open dense subset of the space of initial endowments, the equilibrium price vector is an infinitely differentiable function of the initial endowments.

In this paper, we consider a pure exchange economy with a continuum of agents whose preferences are represented by linear utility functions. Unlike Mertens (1995) who is interested in a somewhat different equilibrium concept, we deal with conventional Walrasian equilibrium. Our aim is to study how the equilibrium prices vary with respect to preferences and initial endowments. Actually, our main objective is to see if the above quoted result of Bonnisseau and Jofré (1994) holds in continuum economies.

We first state the model and give sufficient conditions of an increasing strength for existence, uniqueness and continuity of equilibrium prices. Next, we provide an explicit formula which will be used in the following to compute the equilibrium price vector around a well-defined point where it is known. Finally, if we restrict ourselves to economies wi h essentially bounded initial endowments and if we assume that, from the point of view of preferences, there is only a finite number of types of agents, we show that on an open dense subset of the space of initial endowments, the equilibrium price vector is an infinitely differentiable function of the initial endowments.

\section{The Model. Existence, uniqueness and con- tinuity of equilibrium prices}

We consider in this paper linear exchange economies with a positive finite number $\ell$ of commodities and an atomless positive, bounded measure space $(I, \mathcal{A}, \mu)$ of agents. For simplicity, we assume that $I$ is the real interval $[0,1], \mathcal{A}$ the Borel $\sigma$-algebra of subsets of $I$ and $\mu$ the Lebesgue measure. The consumption set of each agent $t \in I$ is $X_{t}=\mathbb{R}_{+}^{\ell}$, his initial endowment is $\omega(t) \in \mathbb{R}_{+}^{\ell}$ and his preference relation is represented by a linear utility function $U_{t}: X_{t} \rightarrow \mathbb{R}$, defined as $U_{t}(x)=a(t) \cdot x=\sum_{h=1}^{\ell} a_{h}(t) x_{h}$, for some given vector $a(t) \in \mathbb{R}_{+}^{\ell}$. If we denote by $\omega: I \rightarrow \mathbb{R}_{+}^{\ell}$ and by $a: I \rightarrow \mathbb{R}_{+}^{\ell}$ the functions that respectively associate to each agent his initial endowment and the vector $a(t)$, a linear exchange economy is defined by the following list of data $\mathcal{E}(a, \omega)=\left((I, \mathcal{A}, \mu),(a(t), \omega(t))_{t \in I}\right)$.

We will consider on $\mathcal{E}(a, \omega)$ the following assumptions that we adapt from 
Cornet (1989):

(A.1) The map $a: I \rightarrow \mathbb{R}_{+}^{\ell}$ that associates to each agent $t \in I$ the vector $a(t) \in \mathbb{R}_{+}^{\ell}$ which defines his utility function is measurable and $a(t) \neq 0$ for almost every $t \in I$.

(A.2) The map $\omega: I \rightarrow \mathbb{R}_{+}^{\ell}$ that associates to each agent his initial endowment is integrable and $\omega(t) \neq 0$ for almost every $t \in I$.

(A.3) For every commodity $h \in\{1, \cdots, \ell\}$, there exist measurable subsets of agents $A, B \subset I$, with $\mu(A)>0, \mu(B)>0$ such that $a_{h}(t)>0$ for almost every $t \in A$ and $\omega_{h}(t)>0$ for almost every $t \in B$, where $a_{h}(t)$ and $\omega_{h}(t)$ denote the $h$-th coordinate of $a(t)$ and $\omega(t)$ respectively.

(A.4) If $A \in \mathcal{A}$ is such that for some $H \subset\{1, \cdots, \ell\}, \omega_{h}(t)=0$ for almost every $t \in A$, for all $h \in H$, and $a_{k}(t)=0$ for almost every $t \notin A$, for all $k \notin H$, then either $\mu(A)=0$ or $\mu(A)=1$.

(A.5) There exists $J \in \mathcal{A}$ with $\mu(J)>0$ such that $\omega(t)>>0$ for almost every $t \in J$.

(A.6) $a(t)>>0$ for almost every $t \in I$.

(A.7) $\omega(t)>0$, for almost every $t \in I$.

Assumptions (A.1) and (A.2) are standard and define linear exchange economies with a continuum of agents. When it is made on some $\mathcal{E}(a, \omega)$, Assumption (A.3) is clearly harmless, for if it were violated it would be simply possible to remove the goods $h$ that don't satisfy (A.3). Note that (A.3) guarantees that $\int_{I} \omega(t)>>0$. (A.4) is an irreducibility assumption, first formulated by Gale (1957) for an economy with a finite number of agents ; in view of (A.1) and (A.2), (A.4) is in particular satisfied under (A.6) which guarantees desirability of the commodities as in Aumann (1966). (A.5) (and a fortiori (A.7)) and (A.6) strengthen (A.3) and (A.4).

Recall that an allocation is a $\mu$-integrable function $x: I \rightarrow \mathbb{R}_{+}^{\ell}$. An allocation $x \in L^{1}\left(\mathbb{R}_{+}^{\ell}\right)$ is said to be feasible if $\int_{I} x(t) d \mu \leq \int_{I} \omega(t) d \mu$. A quasiequilibrium is a pair $(p, x) \in \mathbb{R}_{+}^{\ell} \times L^{1}\left(\mathbb{R}_{+}^{\ell}\right)$, consisting of a nonzero price system $p$ and a feasible allocation $x$, such that for almost every $t \in I, p \cdot x(t) \leq p \cdot \omega(t)$ and $a(t) \cdot z>a(t) \cdot x(t) \Rightarrow p \cdot z \geq p \cdot \omega(t)$. This quasiequilibrium is a competitive (or Walrasian) equilibrium if for almost every $t \in I, x(t)$ maximizes $a(t) \cdot z$ in $B_{t}(p)=\left\{z \in \mathbb{R}_{+}^{\ell} \mid p \cdot z \leq p \cdot \omega(t)\right\}$.

Before establishing an existence and uniqueness result, we prove a lemma for which we need some additional notation. Given a price system $p \in \mathbb{R}_{++}^{\ell}$, let us denote by $H_{t}(p)$ the commodity subset defined as

$$
H_{t}(p)=\left\{h \in\{1, \ldots, \ell\} \mid \frac{a_{h}(t)}{p_{h}}=\max _{k} \frac{a_{k}(t)}{p_{k}}\right\} .
$$

Let us denote by $d_{t}(p, \omega(t))$ the demand correspondence of the agent $t \in I$, by $v_{t}$ 
his indirect utility function. Finally, let $e^{h} \in \mathbb{R}_{+}^{\ell}$ be the vector whose coordinates are equal to 0 , except the $h$-th which is equal to 1 .

Lemma 2.1 Let $\mathcal{E}(a, \omega)$ be a linear exchange economy. Then for all $p, q \in \mathbb{R}_{++}^{\ell}$ and for all $t \in I$, such that $a(t) \neq 0$ and $\omega(t) \neq 0$, the following statements hold:

i) $d_{t}(p, \omega(t))=\operatorname{co}\left(\left\{\frac{p \cdot \omega(t)}{p_{h}} e^{h} \mid h \in H_{t}(p)\right\}\right)$, where for $X \subset \mathbb{R}^{\ell}, \operatorname{co}(X)$ denotes the convex hull of $X$

ii) $v_{t}(p)=p \cdot \omega(t) \max _{h} \frac{a_{h}(t)}{p_{h}}$

iii) Let $\alpha \in] 0,1\left[\right.$ and let $r^{\alpha} \in \mathbb{R}_{++}^{\ell}$ be defined by $r_{h}^{\alpha}=\left(p_{h}\right)^{\alpha}\left(q_{h}\right)^{1-\alpha}$ for every $h \in\{1, \cdots, \ell\}$. Then, for all $t \in I, \quad$ it is satisfied that $v_{t}\left(r^{\alpha}\right) \leq \alpha v_{t}(p)+(1-\alpha) v_{t}(q)$

iv) Furthermore, the inequality is strict whenever $v_{t}(p) \neq v_{t}(q)$

Proof. $d_{t}(p, \omega(t))$ is the nonempty set of solutions and $v_{t}(p)$ the value of the linear programming problem

$$
\max a(t) \cdot z \text { subject to } z \geq 0 \text { and } p \cdot z \leq p \cdot \omega(t) .
$$

i) follows from necessary and sufficient conditions for optimality of any element belonging to $d_{t}(p, \omega(t))$.

ii) follows from $v_{t}(p)=a(t) \cdot x$ for any $x \in d_{t}(p, \omega(t))$.

For every $t \in I$, let us now consider the function $V_{t}: \mathbb{R}^{\ell} \rightarrow \mathbb{R}$ defined by $V_{t}(q)=v_{t}\left(\exp \left(q_{1}\right), \ldots, \exp \left(q_{\ell}\right)\right)$. By definition of $V_{t}$ one has that $V_{t}(q)=$ $\max _{h}\left[\sum_{k=1}^{\ell} \omega_{k}(t) a_{h}(t) \exp \left(q_{k}-q_{h}\right)\right]$ for every $q \in \mathbb{R}^{\ell}$. One deduces that $V_{t}$ is convex as the supremum of convex functions. iii) follows from the convexity of $V_{t}$.

The proof of $i v$ ) can be found in Cornet (1989) and lies on the simple observation that for all $\left.x, y \in \mathbb{R}_{+}, \alpha \in\right] 0,1\left[\right.$, it is verified that $\alpha x+(1-\alpha) y \geq x^{\alpha} y^{1-\alpha}$, with a strict inequality if $x \neq y$.

Next, we state the existence and uniqueness of equilibrium prices in linear exchange economies with an atomless agent space. Proposition 2.1 extends Gale (1976) and proves the existence of equilibria and the uniqueness of equilibrium utility levels for almost every consumer. Proposition 2.2 extends Theorem 3 in Cornet (1989).

Proposition 2.1 Under the assumptions (A.1)-(A.4), the atomless linear exchange economy $\mathcal{E}(a, \omega)$ has an equilibrium $(x, p)$ such that $p>>0$ and $\int_{I} x(t) d \mu=$ $\int_{I} \omega(t) d \mu$.

Furthermore, if $(x, p)$ and $(y, q)$ are two equilibria of $\mathcal{E}(a, \omega)$, let us define for $\alpha \in] 0,1\left[, z^{\alpha}=\alpha x+(1-\alpha) y\right.$, and for every $h r_{h}^{\alpha}=\left(p_{h}\right)^{\alpha}\left(q_{h}\right)^{1-\alpha}$. Then $\left(z^{\alpha}, r^{\alpha}\right)$ is also an equilibrium of $\mathcal{E}$ and for almost every $t \in I, v_{t}(p)=v_{t}\left(r^{\alpha}\right)=v_{t}(q)$. 
Proof. The proof of the fist assertion is as in Mertens (1995) (see lemma 3). Let $a^{n}(t)=a(t)+n^{-1}(1, \ldots, 1)$ and consider the sequence of perturbed economies $\mathcal{E}_{n}=\left((I, \mathcal{A}, \mu),\left(a^{n}(t), \omega(t)\right)_{t \in I}\right) .\left(\mathcal{E}_{n}\right)$ satisfies all assumptions of Aumann (1966) for the existence of a competitive equilibrium $\left(p^{n}, x^{n}\right)$, with $p^{n}>>0$, normalized in the unit simplex, and $\int_{I} x^{n}(t) d \mu=\int_{I} \omega(t) d \mu$. W.l.o.g, one can assume that $p^{n} \rightarrow p \neq 0$. Then, by Fatou's lemma (see for example Hildenbrand (1974) or Artstein (1979)), there exists an integrable function $x:[0,1] \rightarrow \mathbb{R}_{+}^{\ell}$, such that $\int_{I} x(t) d \mu \leq \int_{I} \omega(t) d \mu$ and $x(t)$ is a limit point of $\left(x^{n}(t)\right)_{n \geq 1}$ for almost every $t \in[0,1]$. It is easy to check that $(x, p)$ is a quasiequilibrium of $\mathcal{E}(a, \omega)$. Then let $A=\{t \in[0,1] \mid p \cdot \omega(t)=0\}$ and $H=\left\{h \mid p_{h}>0\right\}$. In view of $(\mathrm{A} .3), \mu(A)<1$. On the other hand, for almost every $t \notin A, x(t)$ maximizes $a(t) \cdot x$ subject to $x \geq 0$ and $p \cdot x \leq p \cdot \omega(t)$. Hence, $a_{h}(t)=0$ for almost every $t \notin A$ and for all $h \notin H$. It then follows from (A.4) that $\mu(A)=0$ and that $(x, p)$ is an equilibrium. From (A.3), one deduces that $p>>0$ and $\int_{I} x(t) d \mu=\int_{I} \omega(t) d \mu$.

Assume now that $(x, p)$ and $(y, q)$ are two equilibria of $\mathcal{E}$ and let $\alpha \in] 0,1[$. From iii) in lemma 2.1, we deduce that for almost every $t \in I, a(t) \cdot z^{\alpha}(t)=$ $\alpha v_{t}(p)+(1-\alpha) v_{t}(q) \geq v_{t}\left(r^{\alpha}\right)$. From the definition of the indirect utility function, it follows that $r^{\alpha} \cdot z^{\alpha}(t) \geq r^{\alpha} \cdot \omega(t)$. On the other hand, $\int_{I} z^{\alpha}(t) d \mu=\int_{I} \omega(t) d \mu$ and it is easily seen that for almost every $t \in I, r^{\alpha} \cdot z^{\alpha}(t)=r^{\alpha} \cdot \omega(t)$ and $v_{t}\left(r^{\alpha}\right)=a(t) \cdot z^{\alpha}$. Hence $\left(z^{\alpha}, r^{\alpha}\right)$ is an equilibrium of $\mathcal{E}$. In view of $\left.i v\right)$ in Lemma $2.1, v_{t}\left(r^{\alpha}\right)=v_{t}(p)=v_{t}(q)$ for almost every $t \in I$.

Proposition 2.2 Under (A.1)-(A.4), let $(x, p)$ and $(y, q)$ be two equilibria of $\mathcal{E}(a, \omega)$, with $r^{\alpha}$ defined as in proposition 1.1, for $\left.\alpha \in\right] 0,1\left[\right.$. Let also $\left(H_{i}\right)_{i=1}^{k}$ be the partition of $H=\{1, \ldots, \ell\}$ generated by the equivalence relation: $h \sim k \Leftrightarrow$ $q_{h} p_{k}=q_{k} p_{h}$. Then

i) For almost every $t \in I$, there exists $i$ such that $H_{t}\left(r^{\alpha}\right) \subset H_{i}$ and $\omega_{k}(t)=0$ for all $k \notin H_{i}$

ii) Consequently, under (A.5), the equilibrium price vector $p(a, \omega)$ is unique up to the multiplication by a positive scalar

Proof. For every $h, k \in H$ and for every $t \in I$, it is verified that $\alpha \frac{p_{k}}{p_{h}} \omega_{k}(t) a_{h}(t)+$ $(1-\alpha) \frac{q_{k}}{q_{h}} \omega_{k}(t) a_{h}(t) \geq\left(\frac{p_{k}}{p_{h}}\right)^{\alpha}\left(\frac{q_{k}}{q_{h}}\right)^{(1-\alpha)} \omega_{k}(t) a_{h}(t)$. Furthermore, the inequality is strict whenever $\omega_{k}(t)>0, a_{k}(t)>0$ and $\frac{p_{k}}{p_{h}} \neq \frac{q_{k}}{q_{h}}$.

Summing over $k$ such that $\omega_{k}(t)>0$, we get

$$
\alpha p \cdot \omega(t) \frac{a_{h}(t)}{p_{h}}+(1-\alpha)(q \cdot \omega(t)) \frac{a_{h}(t)}{q_{h}} \geq r^{\alpha} \cdot \omega(t) \frac{a_{h}(t)}{r_{h}^{\alpha}} .
$$

Then, applying the second assertion of Proposition 2.1, we get that for almost every $t \in I, H_{t}\left(r^{\alpha}\right) \subset H_{t}(p) \cap H_{t}(q)$, hence $H_{t}\left(r^{\alpha}\right) \subset H_{i}$ for some $i=1, \ldots, k$. Furthermore, if $\omega_{k}(t)>0$ for some $k \notin H_{i}$, it holds true that $\alpha v_{t}(p)+(1-$ $\alpha) v_{t}(q)>v_{t}\left(r^{\alpha}\right)$, a contradiction which proves the second part of $i$ ). 
If $J$ is as in (A.5), then for almost every $t \in J H_{t}\left(r^{\alpha}\right)=H$, which proves $\left.i i\right)$.

In the following, the equilibrium price is normalized so as to belong to the unit-simplex $\Delta$ of $\mathbb{R}^{\ell}$; recall that $\Delta$ is compact. We now restrict ourselves to linear exchange economies that satisfy (A.1)-(A.7). Such an economy can be described as a measurable mapping $\mathcal{E}$ of $(I, \mathcal{A}, \mu)$ into $\mathbb{R}_{++}^{\ell} \times \mathbb{R}_{++}^{\ell}$ such that $\int e \circ \mathcal{E} d \mu \ll \infty$, where $e \cdot d$ enotes the second projection of $\mathbb{R}_{++}^{\ell} \times \mathbb{R}_{++}^{\ell}$ on $\mathbb{R}_{++}^{\ell}$.

The distribution $\alpha=\mu \circ \mathcal{E}^{-1}$ of $\mathcal{E}$ is called preference-endowment distribution. It is known that the equilibrium price $p(\mathcal{E})$ only depends on the preferenceendowment distribution.

Let $\mathcal{D}$ denote the space of probability measures $\alpha$ on $\mathbb{R}_{++}^{\ell} \times \mathbb{R}_{++}^{\ell}$ such that $\int e d \alpha \ll \infty$, endowed with the following metric: given $\alpha_{1}, \alpha_{2} \in \mathcal{D}, \eta\left(\alpha_{1}, \alpha_{2}\right)=$ $\rho\left(\alpha_{1}, \alpha_{2}\right)+\left|\int e d \alpha_{1}-\int e d \alpha_{2}\right|$, where $\rho$ denotes the Prohorov metric. Recall that $\rho$ induces the weak convergence topology on $\mathcal{D}$ and $(\mathcal{D}, \eta)$ is a separable space (see Dierker (1975)).

In the following proposition, we state the continuity of the equilibrium price function in a result where we consider economies as similar if they have similar preference-endowment distributions and similar mean endowments.

Proposition 2.3 The equilibrium price function $\alpha \rightarrow p(\alpha) \in \Delta$ is continuous on $(\mathcal{D}, \eta)$.

Proof. See Hidenbrand (1974), Proposition 4 p. 152.

Corollary 2.1 Let $\mathcal{E}(a, \omega)$ and, for every $n, \mathcal{E}\left(a^{n}, \omega^{n}\right)$ be economies satisfying (A.1)-(A.7) and such that $\left(a^{n}, \omega^{n}\right)$ converges to $(a, \omega)$ almost everywhere, and $\int_{I} \omega^{n}(t) d \mu$ converges to $\int_{I} \omega(t) \dot{a} \mu$. Then $p\left(a^{n}, \omega^{n}\right)$ converges to $p(a, \omega)$.

Proof. Since convergence almost everywhere implies convergence in distribution, one obtain that $\eta\left(\alpha_{\left(a^{n}, \omega^{n}\right)}, \alpha_{(a, \omega)}\right) \rightarrow 0$ when $n \rightarrow \infty$. Use now Proposition 2.3 to obtain that $p\left(a^{n}, \omega^{n}\right)$ converges to $p(a, \omega)$.

However, we can use Fatou's lemma in order to give a direct proof of this result. Let us consider $x^{n}: I \rightarrow \mathbb{R}_{+}^{\ell}$, competitive equilibrium allocations of the economies $\mathcal{E}\left(a^{n}, \omega^{n}\right)$. W.l.o.g., one can assume that $p\left(a^{n}, \omega^{n}\right)$ converge to some $p \in \Delta$ and that $\lim _{n \rightarrow \infty} \int_{I} x^{n}(t) d \mu$ exists. By Fatou's lemma, one concludes that there exists an integrable function $x: I \rightarrow \mathbb{R}_{+}^{\ell}$, such that $\int_{I} x(t) d \mu \leq \lim _{n \rightarrow \infty} \int_{I} x^{n}(t) d \mu \leq \lim _{n \rightarrow \infty} \int_{I} \omega^{n}(t) d \mu=\int_{I} \omega(t) d \mu$ and $x(t)$ is a limit point of $\left(x^{n}(t)\right)_{n \geq 1}$ for almost every $t \in I$. It is easy to check that $(p, x)$ is a quasiequilibrium and, in view of (A.7), a Walrasian equilibrium of the economy $\mathcal{E}(a, \omega)$. Hence $p=p(a, \omega)$. 
Actually, we have just proved that every converging subsequence of $p\left(a^{n}, \omega^{n}\right)$ converges to $p(a, \omega)$. As $\Delta$ is compact, it is enough to guarantee that $p\left(a^{n}, \omega^{n}\right) \rightarrow$ $p(a, \omega)$.

\section{An explicit formula for the equilibrium price vector}

From now and for the remainder of the paper, we fix the measurable mapping $a: I \rightarrow \mathbb{R}_{++}^{\ell}$ which defines individual utility functions, so that the price vector is a function $\omega \rightarrow p^{*}(\omega)$ defined on $L^{1}\left(\mathbb{R}_{++}^{\ell}\right)$.

In this section, we provide the explicit formula which will be used in Section 4 to compute the equilibrium price vector around a point where it is known. In fact, we show that the equilibrium price vector is the unique (up to a multiplicative real factor) positive solution of a linear system. Towards stating the explicit formula, we introduce the following notations.

Let $\mathcal{C}$ be the set of correspondences from the agent set $I$ to the commodity set $\{1, \ldots, \ell\}$, and let $\Omega$ be a subset (to be precised later) of $L^{1}\left(\mathbb{R}_{++}^{\ell}\right)$. We consider the mapping $\Theta$ from $\Omega$ to $\mathcal{C}$ defined as follows : for each $\omega \in \Omega$, the image $\Theta^{\omega}$ is given by $\Theta^{\omega}(t)=H_{t}\left(p^{*}(\omega)\right)$, for every $t \in I$. Let $\tilde{\mathcal{C}}$ be the range of $\Theta$ and, for all $C \in \tilde{\mathcal{C}}$, let us define $\Omega^{C}=\Theta^{-1}(C)=\left\{\omega \in \Omega \mid \Theta^{\omega}=C\right\}$. We fix now $C=\Theta^{\omega} \in \tilde{C}$ and define the following subsets of agents. For each $L \in \mathcal{P}$ (where $\mathcal{P}$ denotes the collection of all nonempty subsets of $\{1, \cdots, \ell\}$ ), $I_{L}=\{t \in I \mid L=C(t)\}$. If $N=\bigcup\left\{I_{L} \mid L \in \mathcal{P}, \mu\left(I_{L}\right)=0\right\}$, note that $\mu(N)=0$, $I=\left(\bigcup\left\{I_{L} \mid L \in \mathcal{P}, \mu\left(I_{L}\right)>0\right\}\right) \bigcup N$ and $I_{L} \cap I_{L^{\prime}}=\emptyset$ if $L \neq L^{\prime}$. So, $\left\{I_{L} \mid L \in\right.$ $\mathcal{P}, \mu(L)>0\} \cup N$ is a finite partition of the agent set $I$. Let us define the nonoriented graph $G_{C}$ as follows. The set of vertices is $\left\{I_{L} \mid L \in \mathcal{P}, \mu\left(I_{L}\right)>0\right\}$, and there exists an edge between $I_{L}$ and $I_{L^{\prime}}$ if and only if $L \cap L^{\prime} \neq \emptyset$. We denote by $\mathcal{I}_{1}^{C}, \cdots, \mathcal{I}_{n}^{C}$ the connected components of $G_{C}$ and by $\mathcal{H}_{1}^{C}, \ldots, \mathcal{H}_{n}^{C}$ the subsets of $\{1, \cdots, \ell\}$, defined by $\mathcal{H}_{j}^{C}=\bigcup_{I_{L} \in \mathcal{I}_{j}^{C}}\{L\}, j=1, \cdots, n$. One readily sees that $\mathcal{H}_{1}^{C}, \cdots, \mathcal{H}_{n}^{C}$ is a partition of the commodity set $\{1, \ldots, \ell\}$. Finally, for each $j \in\{1, \cdots, n\}$, we choose an element $\left(I_{L^{j}}, h^{j}\right) \in \mathcal{I}_{j}^{C} \times \mathcal{H}_{j}^{C}$, such that $h^{j} \in L^{j}$, that is, $h^{j} \in C(t)=H_{t}\left(p^{*}(\omega)\right)$, for all $t \in I_{L^{j}}$.

Next we state a lemma which shows the link between the equilibrium price vector and the finite partition of $I$. For each good $h$, let $S_{h}=\{t \in I \mid h \in C(t)\}$. Observe that $\mu\left(S_{h}\right)>0$ whatever the commodity $h$ may be. Furthermore, $I_{L} \subset$ $S_{h}$ if $h \in L$; if $h \neq h^{\prime}$ and $\left\{h, h^{\prime}\right\} \subset L$, then $I_{L} \subset S_{h} \cap S_{h^{\prime}}$.

Lemma 3.1 There exists a vector $\gamma^{C} \in \mathbb{R}_{++}^{\ell}$ such that for all $\omega \in \Omega^{C}$, for all $j \in\{1, \cdots, n\}$ and for all $h \in \mathcal{H}_{j}^{C}$, it is verified that $p_{h}^{*}(\omega)=\gamma_{h}^{C} p_{h^{j}}^{*}(\omega)$.

Proof. Let us consider $\omega \in \Omega^{C}, j \in\{1, \cdots, n\}$ and $h \in \mathcal{H}_{j}^{C}$. By definition of the 
commodity subsets $\mathcal{H}_{j}^{C}$, there exists $L \in \mathcal{P}$ such that $I_{L} \in \mathcal{I}_{j}^{C}$ and $h \in L$. By definition of connected components, one deduces that there exist $q-1$ elements of $\mathcal{P}, L_{1}, \cdots, L_{q-1}$ and $q$ goods $h_{1}, \cdots, h_{q}$, such that $h_{1} \in L^{j} \cap L_{1}, h_{k} \in L_{k-1} \cap L_{k}$, $k=2, \cdots, q-1$ and $h_{q} \in L_{q-1} \cap L$. Recall that $L=H_{t}\left(p^{*}(\omega)\right)$ for all $t \in I_{L}$. So, by definition of the commodity subsets $H_{t}\left(p^{*}(\omega)\right)$, one deduces that

$$
\begin{array}{ll}
\frac{p_{h^{j}}^{*}(\omega)}{a_{h^{j}}(t)}=\frac{p_{h_{1}}^{*}(\omega)}{a_{h_{1}}(t)} \quad \text { for all } t \in S_{h^{j}} \cap S_{h_{1}} \\
\frac{p_{h_{k}}^{*}(\omega)}{a_{h_{k}}(t)}=\frac{p_{h_{k+1}}^{*}(\omega)}{a_{h_{k+1}}(t)} \quad \text { for all } t \in S_{h_{k}} \cap S_{h_{k+1}} \quad k=2, \ldots, q-1 \\
\frac{p_{h_{q}}^{*}(\omega)}{a_{h_{q}}(t)}=\frac{p_{h}^{*}(\omega)}{a_{h}(t)} \quad \text { for all } t \in S_{h_{q}} \cap S_{h} .
\end{array}
$$

Note that, by construction, $I_{L^{j}} \subset S_{h^{j}} \cap S_{h_{1}}, I_{L_{k}} \subset S_{h_{k}} \cap S_{h_{k+1}}$, for $k=2, \cdots, q-1$ and $I_{L} \subset S_{h_{q}} \cap S_{h}$. Therefore, $\mu\left(S_{h^{j}} \cap S_{h_{1}}\right)>0, \mu\left(S_{h_{k}} \cap S_{h_{k+1}}\right)>0$, for $k=$ $2, \cdots, q-1$ and $\mu\left(S_{h_{q}} \cap S_{h}\right)>0$. Let us consider $t_{q} \in S_{h_{q}} \cap S_{h}, t_{k} \in S_{h_{k}} \cap S_{h_{k+1}}$ for $k=2, \cdots, q-1, t^{j} \in S_{h^{j}} \cap S_{h_{1}}$, and define $\gamma^{C} \in \mathbb{R}_{++}^{\ell}$ as follows

$$
\gamma_{h}^{C}=\frac{a_{h}\left(t_{q}\right)}{a_{h_{q}}\left(t_{q}\right)} \frac{a_{h}\left(t_{q-1}\right)}{a_{h_{q-1}}\left(t_{q-1}\right)} \cdots \frac{a_{h_{2}}\left(t_{1}\right)}{a_{h_{1}}\left(t_{1}\right)} \frac{a_{h_{1}}\left(t^{j}\right)}{a_{h^{j}}\left(t^{j}\right)} .
$$

Hence, from the above equalities, we can conclude that $p_{h}^{*}(\omega)=\gamma_{h}^{C} p_{h^{j}}^{*}(\omega)$.

Let us now define the linear map, $\Gamma^{C}$, from $\mathbb{R}^{\ell}$ to $\mathbb{R}^{n}$, as follows :

$$
\Gamma_{j}^{C}(x)=\sum_{h \in \mathcal{H}_{j}^{C}} \gamma_{h}^{C} x_{h}, \text { for every } x \in \mathbb{R}^{\ell},
$$

where $\Gamma_{j}^{C}(x)$ is the $j$-th component of $\Gamma^{C}(x)$, with $j \in\{1, \cdots, n\}$. Let us also consider the $n \times n$ matrix $A^{C}(\omega)$, whose $i j$-element is defined by

$$
a_{i j}^{C}(\omega)= \begin{cases}\Gamma_{i}^{C}\left(\int_{\left(\mathcal{T}_{i}^{C}\right)^{c}} \omega(t) d \mu\right) & \text { if } j=i \\ -\Gamma_{j}^{C}\left(\int_{\mathcal{T}_{i}^{C}} \omega(t) d \mu\right) & \text { if } j \neq i\end{cases}
$$

where $\left(\mathcal{I}_{i}^{C}\right)^{c}$ is the complement set of $\mathcal{I}_{i}^{C}$. We will denote by $A_{j}^{C}(\omega)$ the $(n-$ 1) $\times(n-1)$ submatrix of $A^{C}(\omega)$ obtained by suppressing the $j$-th column and the $j$-th row.

Lemma 3.2 The rank of $A^{C}(\omega)$ is $n-1$.

Proof. Let us first notice that $A^{C}(\omega)$ is a singular matrix. It is so because the sum of its columns is zero. Let us calculate now the sum of the $k$-th column of the $(n-1) \times(n-1)$ submatrix $A_{j}^{C}(\omega)$.

$$
\sum_{\substack{i=1 \\ i \neq j}}^{n} a_{i k}^{C}(\omega)=\Gamma_{k}^{C}\left(\int_{\left(\mathcal{T}_{k}^{C}\right)^{c}} \omega(t) d \mu-\sum_{\substack{i=1 \\ i \neq j, k}}^{n} \int_{\mathcal{I}_{i}^{C}} \omega(t) d \mu\right)=\Gamma_{k}^{C}\left(\int_{\mathcal{I}_{j}^{C}} \omega(t) d \mu\right)>0 .
$$


So, $A_{j}^{C}(\omega)$ is a matrix with nonpositive nondiagonal terms and a strictly positive sum of columns. The properties of such matrices (called diagonal dominant matrices) are known and used in Leontief's systems (see McKenzie (1960), Gale (1960) or Nikaido (1972)) ; in particular, they are regular and have a nonnegative inverse. Consequently the rank of the matrix $A^{C}(\omega)$ is $n-1$.

Theorem 3.1 For all $\omega \in \Omega^{C}$, the equilibrium price vectors are the positive solutions of the following linear system:

$$
A^{C}(\omega)\left(\begin{array}{c}
p_{h^{1}} \\
\vdots \\
p_{h^{n}}
\end{array}\right)=0
$$

For all $j=1, \cdots, n$, for all $h \in \mathcal{H}_{j}^{C}, h \neq h^{j}, p_{h}=\gamma_{h}^{C} p_{h^{j}}$.

Proof. Consider $\omega \in \Omega^{C}$. Let us first show that $p^{*}(\omega)$ solves the linear system. By lemma 3.1, it is sufficient to prove that

$$
A^{C}(\omega)\left(\begin{array}{c}
p_{h^{1}}^{*}(\omega) \\
\vdots \\
p_{h^{n}}^{*}(\omega)
\end{array}\right)=0
$$

Let us consider an equilibrium allocation $x$ of the economy $\mathcal{E}(\omega)$. Then, for almost all $t \in I$ it is verified that $p^{*}(\omega) \cdot x(t)=p^{*}(\omega) \cdot \omega(t)$. Hence,

$$
\int_{\mathcal{I}_{j}^{C}} p^{*}(\omega) \cdot x(t) d \mu=p^{*}(\omega) \cdot \int_{\mathcal{I}_{j}^{C}} \omega(t) d \mu=\sum_{k=1}^{n} p_{h^{k}}^{*}(\omega) \Gamma_{k}^{C}\left(\int_{\mathcal{T}_{j}^{C}} \omega(t) d \mu\right)
$$

where the last equality comes from Lemma 3.1 and from the definition of the map $\Gamma^{C}$. By Lemma 2.1, one has, for all $j=1, \cdots, n$ and for all $h \in \mathcal{H}_{j}^{C}, x_{h}(t)=0$ if $t \notin \mathcal{I}_{j}^{C}$. So $\int_{\mathcal{I}_{j}^{C}} x_{h}(t) d \mu=\int_{I} \omega_{h}(t) d \mu$, whatever $h \in \mathcal{H}_{j}^{C}$ may be. Furthermore, for all $h \notin \mathcal{H}_{j}^{C}$, it is verifed that $\int_{\mathcal{I}_{j}^{C}} x_{h}(t) d \mu=0$.

It is also verified that

$$
\begin{aligned}
\int_{\mathcal{T}_{j}^{C}} p^{*}(\omega) \cdot x(t) d \mu & =\int_{\mathcal{T}_{j}^{C}} \sum_{h \in \mathcal{H}_{j}^{C}} p_{h}^{*}(\omega) x_{h}(t) d \mu \\
& =\sum_{h \in \mathcal{H}_{j}^{C}} p_{h}^{*}(\omega) \int_{\mathcal{I}_{j}^{C}} x_{h}(t) d \mu \\
& =\sum_{h \in \mathcal{H}_{j}^{C}} p_{h}^{*}(\omega) \int_{I} \omega_{h}(t) d \mu \\
& =p_{h^{j}}^{*}(\omega) \sum_{h \in \mathcal{H}_{j}^{C}} \gamma_{h}^{C} \int_{I} \omega_{h}(t) d \mu \\
& =p_{h^{j}}^{*}(\omega) \Gamma_{j}^{C}\left(\int_{I} \omega(t) d \mu\right) .
\end{aligned}
$$

Hence one has for all $j=1, \cdots, n$,

$$
\sum_{\substack{k=1 \\ k \neq j}}^{n} p_{h^{k}}^{*}(\omega)\left(\int_{\mathcal{I}_{j}^{C}} \omega(t) d \mu\right)=p_{h^{j}}^{*}(\omega) \Gamma_{j}^{C}\left(\int_{C} \omega(t) d \mu-\int_{\mathcal{I}_{j}^{C}} \omega(t) d \mu\right)
$$


Since $\int_{I} \omega(t) d \mu-\int_{\mathcal{T}_{j}^{C}} \omega(t) d \mu=\int_{\left(\mathcal{T}_{j}^{C}\right)^{c}} \omega(t) d \mu$, one concludes that $p^{*}(\omega)$ solves the linear system.

It remains to show that, if $p$ is a positive solution of the linear system, then $p$ is an equilibrium price. By Lemma 3.2, we know that the rank of the matrix $A^{C}(\omega)$ is $n-1$ and so the solution set of the linear system is a one dimensional subspace of $\mathbb{R}^{\ell}$ which contains the half line $\left\{\lambda p^{*}(\omega) \mid \lambda>0\right\}$. Hence, the solution set is $\mathbb{R} p^{*}(\omega)$ and every positive solution is an equilibrium price vector.

Corollary 3.1 Assume that we choose a good $h^{j}$ as numeraire, with $1 \leq j \leq n$. Then, for all $\omega \in \Omega^{C}$, the price vector $p^{*}(\omega)$ comes determinated by the following equations :

$$
p_{h^{j}}^{*}(\omega)=1,\left(\begin{array}{c}
p_{h^{1}}^{*}(\omega) \\
\vdots \\
p_{h^{j-1}}^{*}(\omega) \\
p_{h^{j+1}}^{*}(\omega) \\
\vdots \\
p_{h^{n}}^{*}(\omega)
\end{array}\right)=-\left(A_{j}^{C}(\omega)\right)^{-1}\left(\begin{array}{c}
a_{1 j}^{C}(\omega) \\
\vdots \\
a_{j-1 j}^{C}(\omega) \\
a_{j+1 j}^{C}(\omega) \\
\vdots \\
a_{n j}^{C}(\omega)
\end{array}\right)
$$

and for all $j=1, \cdots, n$, for all $h \in \mathcal{H}_{j}^{C}, h \neq h^{j}$,

$$
p_{h}^{*}(\omega)=\gamma_{h}^{C} p_{h^{j}}^{*}(\omega)
$$

Furthermore, all the elements of the matrix $\left(A_{j}^{C}(\omega)\right)^{-1}$ are non negative.

Proof. By the proof of Lemma 3.2, one obtains that the matrix $A_{j}^{C}(\omega)$ has full rank. So, the given equation system has a unique solution. By Theorem 3.1, we know that the equilibrium price vector $p^{*}(\omega)$, with $p_{h j}^{*}(\omega)=1$ is a solution. Therefore, $p^{*}(\omega)$ is the unique solution of the system. Finally, as already noticed, the elements of the inverse matrix $\left(A_{j}^{C}(\omega)\right)^{-1}$ are non negative.

\section{A generic property of equilibrium prices}

Let us now assume that the map $a: I \rightarrow \mathbb{R}_{++}^{\ell}$, which defines the utility functions of the agents, is a given simple function. More precisely, $a(t)=\sum_{i=1}^{s} a_{i} \chi_{A_{i}}(t)$, where for all $i \in\{1, \cdots, s\}, A_{i} \in \mathcal{A}, a_{i} \in \mathbb{R}_{++}^{\ell}, \chi_{A_{i}}$ is the characteristic function of $A_{i}$, and $A_{i} \cap A_{j}=\emptyset$ if $i \neq j, I=\bigcup_{i=1}^{s} A_{i}$. In other words, from the point of view of preferences, there exists in the economy a finite number $s$ of agents. Note that the subset of simple maps is dense in $\left(L^{1}\left(\mathbb{R}_{++}^{\ell}\right),\|\cdot\|_{1}\right)$ and in $\left(L^{\infty}\left(\mathbb{R}_{++}^{\ell}\right), \| \cdot\right.$ $\left.\|_{\infty}\right)$.

Moreover, we restrict ourselves to initial endowments $\omega$ belonging to $L^{\infty}\left(\mathbb{R}_{++}^{\ell}\right)$. Note that it follows from the explicit computation of the equilibrium demand in Lemma 2.1 that the corresponding equilibrium allocations belong to $L^{\infty}\left(\mathbb{R}_{+}^{\ell}\right)$. 
Definition 4.1 The economy $\mathcal{E}(a, \omega)$ is said to be regular if there exists a Walrasian equilibrium allocation $x: I \rightarrow \mathbb{R}_{+}^{\ell}$ belonging to $L^{\infty}\left(\mathbb{R}_{+}^{\ell}\right)$, and a positive number $b_{x}>0$, such that for almost all $t \in I$ and for all $h \in H_{t}\left(p^{*}(\omega)\right)$, it is verified that $x_{h}(t) \geq b_{x}$.

Let us denote by $\Omega$ the set of initial endowments $\omega \in L^{\infty}\left(\mathbb{R}_{++}^{\ell}\right)$ such that the economy $\mathcal{E}(a, \omega)$ is regular. In what follows, we prove that for each $\omega \in \Omega$ there exists a neighbourhood of $\omega$ included in $\Omega$ where the equilibrium prices map $p^{*}$ is an infinitely differentiable function of the initial endowments. In order to obtain such a result, we state some notations and prove, as technical lemmas, some intermediate claims.

Consider an arbitrary but fixed $\bar{\omega} \in \Omega$. For each $i \in\{1, \cdots, s\}$, let $H_{i}$ be the set of goods $H_{t}\left(p^{*}(\bar{\omega})\right)$, whoever agent $t \in A_{i}$ may be. Let $\beta: I \rightarrow \mathbb{R}_{++}$be the map defined by $\beta(t)=\min \left\{\frac{p_{h}^{*}(\bar{\omega})}{a_{h}(t)} \mid h \in\{1, \cdots, \ell\}\right\}$. For each $i \in\{1, \cdots, s\}$, let $\beta_{i}$ denote the real number $\beta(t)$, whoever agent $t \in A_{i}$ may be. Using the notations of the previous section, state $C=\Theta^{\bar{\omega}}$. We now consider the following linear spaces

$$
\begin{gathered}
\mathcal{M}=\left\{x \in L^{\infty}\left(\mathbb{R}^{\ell}\right) \mid x_{h}(t)=0 \text { if } h \notin H_{t}\left(p^{*}(\bar{\omega})\right), \text { for almost all } t \in I\right\}, \\
\mathcal{L}=\left\{(f, y) \in L^{\infty}(\mathbb{R}) \times \mathbb{R}^{\ell} \mid \int_{\mathcal{T}_{j}^{C}} \beta(t) f(t) d \mu(t)=\sum_{h \in \mathcal{H}_{j}^{C}} p^{*}(\bar{\omega}) y_{h}, \text { for all } j=1, \cdots, n\right\},
\end{gathered}
$$

and the following mappings $\varphi: L^{\infty}\left(\mathbb{R}^{\ell}\right) \rightarrow L^{\infty}(\mathbb{R}) \times \mathbb{R}^{\ell}, \psi: L^{\infty}\left(\mathbb{R}_{++}^{\ell}\right) \rightarrow$ $L^{\infty}(\mathbb{R}) \times \mathbb{R}_{++}^{\ell}$, defined by

$$
\begin{gathered}
\varphi(x)=\left((a(t) \cdot x(t))_{t \in I}, \int_{I} x(t) d \mu(t)\right), \\
\psi(\omega)=\left(\left(v_{t}\left(p^{*}(\omega), \omega(t)\right)_{t \in I}, \int_{I} \omega(t) d \mu(t)\right) .\right.
\end{gathered}
$$

It is worth noticing that $\mathcal{M}$ and $\mathcal{L}$ are closed (in $L^{\infty}\left(\mathbb{R}^{\ell}\right)$ and $L^{\infty}(\mathbb{R}) \times \mathbb{R}^{\ell}$ respectively), that $\mathcal{M}$ contains the equilibrium allocations of $\mathcal{E}(a, \bar{\omega})$ and that $x \in L^{\infty}\left(\mathbb{R}_{+}^{\ell}\right)$ is an equilibrium allocation of $\mathcal{E}(a, \omega)$ if and only if $\varphi(x)=\psi(\omega)$.

\section{Lemma $4.1 \varphi(\mathcal{M}) \subset \mathcal{L}$.}

Proof. Consider $x \in \mathcal{M}$ and $\varphi(x)=(f, y)$. By definition of $\mathcal{M}$ and $\varphi$,

$$
f(t)=a(t) \cdot x(t)=\sum_{h \in H_{t}\left(p^{*}(\bar{\omega})\right)} a_{h}(t) x_{h}(t)=\frac{1}{\beta(t)} \sum_{h \in H_{t}\left(p^{*}(\bar{\omega})\right)} p_{h}^{*}(\bar{\omega}) x_{h}(t) .
$$

Noticing that for all $j=1, \cdots, n$, for almost all $t \notin \mathcal{I}_{j}^{C}, x_{h}(t)=0$, if $h \notin \mathcal{H}_{j}^{C}$, one deduces that for all $j$ and for all $h \in \mathcal{H}_{j}^{C}$,

$$
y_{h}=\int_{I} x_{h}(t) d \mu(t)=\int_{T_{j}^{C}} x_{h}(t) d \mu(t) .
$$


So, for all $j=1, \cdots, n$, we can conclude that

$$
\begin{aligned}
\int_{\mathcal{T}_{j}^{C}} \beta(t) f(t) d \mu(t) & =\int_{\mathcal{T}_{j}^{C}} \sum_{h \in H_{t}\left(p^{*}(\bar{\omega})\right)} p_{h}^{*}(\bar{\omega}) x_{h}(t) d \mu(t) \\
& =\sum_{h \in \mathcal{H}_{j}^{C}} p_{h}^{*}(\bar{\omega}) \int_{\mathcal{T}_{j}^{C}} x_{h}(t) d \mu(t) \\
& =\sum_{h \in \mathcal{H}_{j}^{C}} p_{h}^{*}(\bar{\omega}) y_{h} .
\end{aligned}
$$

\section{Lemma $4.2 \varphi$ is continuous.}

Proof. Let $\varphi_{1}(x)(t)=a(t) \cdot x(t)$, and $\varphi_{2}(x)=\int_{I} x(t) d \mu(t)$. The map $\varphi_{1}$ : $L^{\infty}\left(\mathbb{R}^{\ell}\right) \rightarrow L^{\infty}(\mathbb{R})$ is continuous because $x \in L^{\infty}\left(\mathbb{R}^{\ell}\right)$ and $a$ is a simple function. On the other hand, if $\left\|x_{n}-x\right\|_{\infty} \rightarrow 0$, then there exists $A \in \mathcal{A}$, such that $\mu(I \backslash A)=0$, and $\dot{x}_{n}$ converges uniformly to $x$ on $A$. So, $\varphi_{2}: L^{\infty}\left(\mathbb{R}^{\ell}\right) \rightarrow \mathbb{R}$ is also a continuous map. Therefore $\varphi=\left(\varphi_{1}, \varphi_{2}\right)$ is continuous.

Lemma $4.3 \varphi(\mathcal{M})=\mathcal{L}$.

Proof. Let us denote by $\mathcal{F}_{0}$ the following set

$$
\mathcal{F}_{0}=\left\{f \in L^{\infty}(\mathbb{I R}) \mid \int_{A_{i}} f(t) d \mu(t)=0 \text { for all } i \in\{1, \cdots, s\}\right\}
$$

and for each $i=1, \cdots, s$, let $\mathcal{F}_{i}$ denote the set defined as follows

$$
\mathcal{F}_{i}=\left\{f \in L^{\infty}(\mathbb{R}) \mid \int_{A_{i}} f(t) d \mu(t)=\mu\left(A_{i}\right), \int_{A_{i^{\prime}}} f(t) d \mu(t)=0 \text { for all } i^{\prime} \neq i\right\} .
$$

Let $\mathcal{L}_{0}$ be the set defined by $\mathcal{L}_{0}=\left\{(f, 0) \in L^{\infty}(\mathbb{R}) \times \mathbb{R}^{\ell} \mid f \in \mathcal{F}_{0}\right\}$. For each $j=1, \cdots, n$, let $\mathcal{L}^{j}, \mathcal{L}_{j}$ denote the sets defined by

$$
\begin{aligned}
& \mathcal{L}^{j}=\left\{\left(p_{h}^{*}(\bar{\omega}) \frac{1}{\mu\left(A_{i}\right)} f_{i}, \beta_{i} e^{h}\right) \mid A_{i} \subset \mathcal{I}_{j}^{C}, h \in H_{i} \text { and } f_{i} \in \mathcal{F}_{i}\right\} \\
& \mathcal{L}_{j}=\left\{\left(\frac{1}{\mu\left(A_{i}\right)} f_{i}-\frac{\beta_{i}}{\beta_{k} \mu\left(A_{k}\right)} f_{k}, 0\right) \mid \begin{array}{c}
A_{i}, A_{k} \subset \mathcal{I}_{j}^{C}, A_{i} \neq A_{k}, \\
f_{i} \in \mathcal{F}_{i}, \text { and } f_{k} \in \mathcal{F}_{k}
\end{array}\right\} .
\end{aligned}
$$

It is easy to check that the sets $\mathcal{L}_{0}, \mathcal{L}^{j}$, and $\mathcal{L}_{j}$ are included in $\mathcal{L}$. Consider $\mathcal{G}=\mathcal{L}_{0} \bigcup\left(\bigcup_{i=1}^{n} \mathcal{L}^{j}\right) \bigcup\left(\bigcup_{i=1}^{n} \mathcal{L}_{j}\right)$. It is also easy to check that $\mathcal{G}$ generates $\mathcal{L}(\mathrm{a}$ proof of this claim is given in the appendix). As $\varphi$ is a linear function, it is enough to prove that $\mathcal{G} \subset \varphi(\mathcal{M})$.

Let $(f, 0)$ belong to $\mathcal{L}_{0}$. For each $i \in\{1, \cdots, s\}$, consider a commodity $h(i) \in$ $H_{i}$. Define $x_{h(i)}(t)=\frac{f(t)}{a_{h(i)}(t)}$, and $x_{h}(t)=0$ if $h \neq h(i)$, for all $t \in A_{i}$. By construction, $x \in \mathcal{M}$ and $\varphi(x)=(f, 0)$. 
Consider now $(f, y) \in \mathcal{L}^{j}$ for some $j \in\{1, \cdots, n\}$. That is, $f=p_{h}^{*}(\bar{\omega}) \frac{1}{\mu\left(A_{i}\right)} f_{i}$, and $y=\beta_{i} e^{h}$, with $h \in H_{i}, A_{i} \subset \mathcal{I}_{j}^{C}$, and $f_{i} \in \mathcal{F}_{i}$. So, $\beta_{i}=p^{*}(\bar{\omega})\left(a_{i}\right)_{h}$. For each $i^{\prime} \neq i$, let us consider $h\left(i^{\prime}\right) \in H_{i^{\prime}}$, and define $x: I \rightarrow \mathbb{R}^{\ell}$ as follows

$$
x_{k}(t)=\left\{\begin{array}{l}
0 \quad \text { if }\left\{\begin{array}{l}
\text { either } t \in A_{i^{\prime}}, i^{\prime} \neq i \text { and } k \neq h\left(i^{\prime}\right), \\
\text { or } t \in A_{i} \text { and } k \neq h
\end{array}\right. \\
p_{h}^{*}(\bar{\omega}) \frac{1}{\mu\left(A_{i}\right) a_{h\left(i^{\prime}\right)}(t)} f_{i} \text { if } t \in A_{i^{\prime}}, i^{\prime} \neq i \text { and } k=h\left(i^{\prime}\right) . \\
p_{h}^{*}(\bar{\omega}) \frac{1}{\mu\left(A_{i}\right) a_{h}(t)} f_{i} \text { if } t \in A_{i} \text { and } k=h
\end{array}\right.
$$

By construction, $x \in \mathcal{M}$ and $\varphi(x)=(f, y)$.

Finally, let us consider $(f, 0) \in \mathcal{L}_{j}$ for some $j \in\{1, \ldots, n\}$. That is, $f=$ $\frac{1}{\mu\left(A_{i}\right)} f_{i}-\frac{\beta_{i}}{\beta_{p} \mu\left(A_{p}\right)} f_{p}$, with $A_{i}, A_{p} \subset \mathcal{I}_{j}^{C}, A_{p} \neq A_{i}, f_{i} \in \mathcal{F}_{i}$, and $f_{p} \in \mathcal{F}_{p}$. Two possibilities can now occur, namely, $H_{i}=H_{p}$ or $H_{i} \neq H_{p}$.

Suppose $H_{i}=H_{p}$. For each $i^{\prime} \neq i, p$, let us consider $h\left(i^{\prime}\right) \in H_{i^{\prime}}$ and a fixed $h \in H_{i}=H_{p}$. Define $x: I \rightarrow \mathbb{R}^{\ell}$ as follows

$$
x_{k}(t)=\left\{\begin{array}{l}
0 \quad \text { if }\left\{\begin{array}{l}
\text { either } t \in A_{i^{\prime}}, i^{\prime} \neq i, p \text { and } k \neq h\left(i^{\prime}\right), \\
\text { or } t \in A_{i} \cup A_{p} \text { and } k \neq h
\end{array}\right. \\
\frac{f(t)}{a_{h\left(i^{\prime}\right)}(t)} \text { if } t \in A_{i^{\prime}}, i \neq i, p \text { and } k=h\left(i^{\prime}\right) \\
p_{h}^{*}(\bar{\omega}) \frac{f(t)}{a_{h}(t)} \text { if } t \in A_{i} \cup A_{p} \text { and } k=h
\end{array}\right.
$$

By construction, one concludes that $x \in \mathcal{M}$ and $\varphi(x)=(f, 0)$.

Suppose $H_{i} \neq H_{p}$. Consider $h \in H_{i}$ and $h_{p} \in H_{p}, h_{p} \neq h$. As in the proof of Lemma 3.1, there exist $q-1$ elements of $\mathcal{P}, L_{1}, \cdots, L_{q-1}$ and $q$ goods $h_{1}, \cdots, h_{q}$, such that $h_{1} \in H_{i_{j}} \cap L_{1}, h_{k} \in L_{k-1} \cap L_{k}, k=2, \cdots, q-1$ and $h_{q} \in L_{q-1} \cap H_{i}$. For each $k=1, \cdots, q-1$, let $A_{i(k)}$ be a subset of $I_{L_{k}}$, that is $H_{i(k)}=L_{k}$. By definition of the commodity subsets $H_{t}\left(p^{*}(\bar{\omega})\right)$, one has

$$
\begin{array}{ll}
\frac{p_{h}^{*}(\bar{\omega})}{a_{h}(t)}=\frac{p_{h_{1}}^{*}(\bar{\omega})}{a_{h_{1}}(t)} \quad \text { for all } t \in A_{i} \\
\frac{p_{h_{k}}^{*}(\bar{\omega})}{a_{h_{k}}(t)}=\frac{p_{h_{k+1}}^{*}(\bar{\omega})}{a_{h_{k+1}}(t)} \quad \text { for all } t \in A_{i(k)} \quad k=2, \cdots, q-1 \\
\frac{p_{h_{q}}^{*}(\bar{\omega})}{a_{h_{q}}(t)}=\frac{p_{h_{p}}^{*}(\bar{\omega})}{a_{h_{p}}(t)} \quad \text { for all } t \in A_{p} .
\end{array}
$$


Recalling that $\varphi$ is a linear mapping and $\mathcal{L}^{j} \subset \varphi(\mathcal{M}, j=1, \cdots, n$, one deduces that there exist $x^{k}, \bar{x}^{k} \in \mathcal{M}, k=1, \cdots, q$, such that

$$
\begin{aligned}
& \varphi\left(x^{1}\right)=\left(\left(a_{i}\right)_{h_{1}} \frac{1}{\mu\left(A_{i}\right)} f_{i_{j}}, e^{h_{1}}\right) \\
& \varphi\left(\bar{x}^{k}\right)=\left(\left(a_{i(k)}\right)_{h_{k}} \frac{1}{\mu\left(A_{i(k)}\right)} \chi_{A_{i(k)}}, e^{h_{k}}\right) ; \quad k=1, \cdots, q-1 \\
& \varphi\left(x^{k}\right)=\left(\left(a_{i(k-1)}\right)_{h_{k}} \frac{1}{\mu\left(A_{i(k-1)}\right)} \chi_{A_{i(k-1)}}, e^{h_{k}}\right) ; \quad k=2, \cdots, q \\
& \varphi\left(\bar{x}^{q}\right)=\left(\left(a_{p}\right)_{h_{q}} \frac{1}{\mu\left(A_{p}\right)} f_{p}, e^{h_{q}}\right) .
\end{aligned}
$$

Let us denote $\alpha_{k}=\prod_{r=1}^{k} \frac{\left(a_{i(r)}\right)_{h_{r}}}{\left(a_{i(r)}\right)_{h_{r+1}}}$, for all $k=1, \cdots, q-1$. Let us now consider the map $x: I \rightarrow \mathbb{R}^{\ell}$, defined by $x=x^{1}-\bar{x}^{1}+\sum_{k=1}^{q-1} \alpha_{k}\left(x^{k+1}-\bar{x}^{k+1}\right)$.

By a simple computation,

$$
\begin{aligned}
\varphi(x) & =\left(\left(a_{i_{j}}\right)_{h_{1}} \frac{1}{\mu\left(A_{i_{j}}\right)} f_{i_{j}}-\alpha_{q-1}\left(a_{i}\right)_{h_{q}} \frac{1}{\mu\left(A_{i}\right)} f_{i}, 0\right) \\
& =\left(a_{i}\right)_{h_{1}}\left(\frac{1}{\mu\left(A_{i}\right)} f_{i}-\frac{\beta_{i}}{\beta_{i} \mu\left(A_{p}\right)} f_{p}, 0\right) .
\end{aligned}
$$

Lemma $4.4 \psi$ is a continuous function.

Proof. Denote $\psi=\left(\psi_{1}, \psi_{2}\right)$. The map $\psi_{2}$ is continuous (see Lemma 4.2). Recall that $v_{t}\left(p^{*}(\omega), \omega(t)\right)=p^{*}(\omega) \cdot \omega(t) \max _{h} \frac{a_{h}(t)}{p_{h}^{*}(\omega)}=p^{*}(\omega) \cdot \omega(t) \frac{a_{h}(t)}{p_{h}^{*}(\omega)}$ with $h \in$ $H_{t}\left(p^{*}(\omega)\right)$. Note that the continuity of the equilibrium price vector mapping obtained in Corollary 2.1 implies that $p^{*}$ is a continuous function with respect to the initial endowments $\omega \in L^{\infty}\left(\mathbb{R}_{++}^{\ell}\right)$. Then if $\left\|\omega^{\nu}-\omega\right\|_{\infty} \rightarrow 0$, as $a$ is a simple function, one has for large enough $\nu, H_{t}\left(p^{*}\left(\omega^{\nu}\right)\right) \subset H_{t}\left(p^{*}(\omega)\right)$ and $v_{t}\left(p^{*}\left(\omega^{\nu}\right), \omega^{\nu}(t)\right)=p^{*}\left(\omega^{\nu}\right) \cdot \omega^{\nu}(t) \frac{a_{h}(t)}{p_{h}^{*}\left(\omega^{\nu}\right)}$ with $h \in H_{t}\left(p^{*}(\omega)\right)$. It is now obvious that $v\left(\omega^{\nu}\right)=\left(v_{t}\left(p^{*}\left(\omega^{\nu}\right), \omega^{\nu}(t)\right)\right)_{t \in I}$ converges to $v(\omega)$ for $\|\cdot\|_{\infty}$

We are ready now to state the main results of this section.

Theorem 4.1 For each $\omega \in \Omega$, there exists a neighbourhood $W$ of $\omega$ included in $\Omega$, such that for all $\omega^{\prime} \in W, H_{t}\left(p^{*}\left(\omega^{\prime}\right)\right)=H_{t}\left(p^{*}(\omega)\right)$, for almost all $t \in I$. In particular, $\Omega$ is an open subset of $L^{\infty}\left(\mathbb{R}_{++}^{\ell}\right)$. 
Proof. Let $\bar{\omega}$ be arbitrary but fixed as in Lemmas 4.1, 4.2, 4.3. As we have already noticed, Corollary 2.1 implies the continuity of the equilibrium prices with respect to the initial endowments. So, there exists a neighbourhood $V$ of $\bar{\omega}$, such that for all $\omega \in V, H_{t}\left(p^{*}(\omega)\right) \subset H_{t}\left(p^{*}(\bar{\omega})\right)$, for almost all $t \in I$. From this, it is readily deduced that for all $\omega \in V$, every equilibrium allocation of $\mathcal{E}(a, \omega)$ belongs to $\mathcal{M}$ and hence $\psi(\omega) \in \varphi(\mathcal{M})=\mathcal{L}$. On the other hand, let

$$
\mathcal{A}=\left\{x \in \mathcal{M} \mid \exists b_{x}, x_{h}(t) \geq b_{x}, \text { for almost all } t \in I \text { and for all } h \in H_{t}\left(p^{*}(\bar{\omega})\right)\right\} \text {. }
$$

Since $\mathcal{E}(a, \bar{\omega})$ is a regular economy, $\mathcal{E}(a, \bar{\omega})$ has an equilibrium allocation $\bar{x} \in \mathcal{A}$ and $\psi(\bar{\omega})=\varphi(\bar{x}) \in \varphi(\mathcal{A})$. Note that $\mathcal{A}$ is an open subset of $\mathcal{M}$. Since $\varphi$ is a continuous linear mapping from $\mathcal{M}$ onto $\mathcal{L}$, it follows from the open mapping theorem (see, for example, Beauzamy (1985) or Köthe (1969) p. 166), that $\varphi(\mathcal{A})$ is open in $\mathcal{L}$. Since $\psi$ is continuous, there exists a neighbourhood $V^{\prime}$ of $\bar{\omega}$ such that $\psi\left(V^{\prime}\right) \cap \mathcal{L} \subset \varphi(\mathcal{A})$. Finally, $\omega \in V \cap V^{\prime} \Rightarrow \psi(\omega) \in \varphi(\mathcal{A})$, that is $\omega \in \Omega$ and $H_{t}\left(p^{*}(\bar{\omega})\right) \subset H_{t}\left(p^{*}(\omega)\right) \subset H_{t}\left(p^{*}(\bar{\omega})\right)$.

Corollary 4.1 For each $\omega \in \Omega$, let $W$ be as in Theorem 4.1. The mapping $\omega^{\prime} \rightarrow p^{*}\left(\omega^{\prime}\right)$ is infinitely differentiable on $W$.

Proof. Using the notations of Section 3, set $C=\Theta^{\omega}$. It follows from Theorem 4.1 that $W$ is included in $\Omega^{C}$. Consequently, the formula given in Corollary 3.1 remains true on $W$. As a continuous linear function of $\omega^{\prime}$, each integral used in the computation of the elements of the matrix $A^{C}\left(\omega^{\prime}\right)$ is an infinitely differentiable function of $\omega^{\prime}$ (see Cartan (1977) for a relevant definition of differentiability on Banach spaces). On $W$, each coordinate of $p^{*}\left(\omega^{\prime}\right)$ is a polynomial function of such integrals.

Theorem $4.2 \Omega$ is a dense subset of $L^{\infty}\left(\mathbb{R}_{++}^{\ell}\right)$.

Proof. Consider $\omega \in L^{\infty}\left(\mathbb{R}_{++}^{\ell}\right)$ and $x \in L^{\infty}\left(\mathbb{R}_{+}^{\ell}\right)$ a Walrasian allocation of the economy $\mathcal{E}(a, \omega)$. For each real number $\varepsilon>0$, let $\omega^{\varepsilon}$ denote the initial endowment map defined by

$$
\omega^{\varepsilon}(t)=\omega(t)+\varepsilon \sum_{h \in H_{t}\left(p^{*}(\omega)\right)} e^{h}
$$

and let $x^{\varepsilon}$ denote the allocation defined by

$$
x^{\varepsilon}(t)=x(t)+\varepsilon \sum_{h \in H_{t}\left(p^{*}(\omega)\right)} e^{h} .
$$

Obviously, $\forall h=1, \cdots, \ell,\left\|\omega_{h}^{\varepsilon}-\omega_{h}\right\|_{\infty}<\varepsilon$. Moreover, $x_{h}^{\varepsilon}(t) \geq \varepsilon$ for almost all $t \in I$ and for all $h \in H_{t}\left(p^{*}(\omega)\right)$. To prove that $\omega^{\varepsilon} \in \Omega$, it suffices to prove that 
$\left(x^{\varepsilon}, p^{*}(\omega)\right)$ is a competitive equilibrium of the economy $\mathcal{E}\left(a, \omega^{\varepsilon}\right)$. Indeed,

$$
\begin{aligned}
v_{t}\left(p^{*}(\omega), \omega^{\varepsilon}(t)\right) & =p^{*}(\omega) \cdot \omega^{\varepsilon}(t) \max _{h} \frac{a_{h}(t)}{p_{h}^{*}(\omega)} \\
& =\left(p^{*}(\omega) \cdot \omega(t)+\varepsilon p^{*}(\omega) \cdot \sum_{h \in H_{t}\left(p^{*}(\omega)\right)} e^{h}\right) \max _{h} \frac{a_{h}(t)}{p_{h}^{*}(\omega)}, \text { and } \\
a(t) \cdot x^{\varepsilon}(t) & =a(t) \cdot x(t)+\varepsilon a(t) \cdot \sum_{h \in H_{t}\left(p^{*}(\omega)\right)} e^{h} \\
& =p^{*}(\omega) \cdot \omega(t) \max _{h} \frac{a_{h}(t)}{p_{h}^{*}(\omega)}+\varepsilon a(t) \cdot \sum_{h \in H_{t}\left(p^{*}(\omega)\right)} e^{h}
\end{aligned}
$$

Finally, note that

$$
\left(\varepsilon p^{*}(\omega) \cdot \sum_{h \in H_{t}\left(p^{*}(\omega)\right)} e^{h}\right) \max _{h} \frac{a_{h}(t)}{p_{h}^{*}(\omega)}=\varepsilon a(t) \cdot \sum_{h \in H_{t}\left(p^{*}(\omega)\right)} e^{h} .
$$

\section{Appendix}

\section{Proof that $\mathcal{G}$ generates $\mathcal{L}$.}

Let $(f, y)$ be an element of $\mathcal{L}$. Let us consider two cases.

First case. Suppose that $y=0$. Let $\alpha_{i}=\int_{A_{i}} f(t) d \mu(t), i=1, \cdots, m$. Since $(f, 0) \in \mathcal{L}$, one has for each connected component $j=1, \cdots, n, \sum_{A_{i} \subset \mathcal{I}_{j}^{\omega}} \alpha_{i} \beta_{i}=0$.

Consider now a fixed connected component $j$. Let $S_{j}=\left\{i \mid A_{i} \subset \mathcal{I}_{j}^{\omega}\right.$, such that $\alpha_{i} \neq$ $0\}$ and $S^{j}=\left\{i \mid A_{i} \subset \mathcal{I}_{j}^{\omega}\right.$, such that $\left.\alpha_{i}=0\right\}$. For for each $i$, such that $A_{i} \subset \mathcal{I}_{j}^{\omega}$, define the following function

$$
f_{i}(t)= \begin{cases}\frac{\mu\left(A_{i}\right)}{\alpha_{i}} f(t) \chi_{A_{i}} & \text { if } \alpha_{i} \neq 0 \\ f(t) \chi_{A_{i}} & \text { if } \alpha_{i}=0\end{cases}
$$

So, if $t \in \mathcal{I}_{j}^{\omega}$, then $f(t)=\sum_{i \in S_{j}} \frac{\alpha_{i}}{\mu\left(A_{i}\right)} f_{i}(t)+\sum_{i \in S^{j}} f(t) \chi_{A_{i}}$.

Note that if $S^{j}=\{1, \cdots, s\}$, the proof is finished because if $\alpha_{i}=0$, then $f_{i} \in \mathcal{F}_{0}$. 
Suppose now that $S_{j} \neq \emptyset$. Then there exist $i, k$, such that $\alpha_{i}, \alpha_{k} \neq 0$. By definition of $\mathcal{L}$, one obtains that $\alpha_{k}=-\frac{1}{\beta_{k}} \sum_{i \neq k} \alpha_{i} \beta_{i}$. Then, $f(t) \chi_{\mathcal{I}_{j}^{\omega}}$ can be written as follows

$$
\begin{aligned}
\left(f \cdot \chi_{\mathcal{I}_{j}^{\omega}}\right)(t) & =\frac{\alpha_{k}}{\mu\left(A_{k}\right)} f_{k}(t)+\sum_{\substack{i \in S_{j} \\
i \neq k}} \frac{\alpha_{i}}{\mu\left(A_{i}\right)} f_{i}(t)+\sum_{i \in S^{j}} f(t) \chi_{A_{i}} \\
& =-\frac{1}{\beta_{k}}\left(\sum_{\substack{i \in S_{j} \\
i \neq k}} \frac{\alpha_{i} \beta_{i}}{\mu\left(A_{k}\right)} f_{k}(t)\right)+\sum_{\substack{i \in S_{j} \\
i \neq k}} \frac{\alpha_{i}}{\mu\left(A_{i}\right)} f_{i}(t)+\sum_{i \in S^{j}} f(t) \chi_{A_{i}} \\
& =\sum_{\substack{i \in S_{j} \\
i \neq k}} \alpha_{i}\left(\frac{1}{\mu\left(A_{i}\right)} f_{i}(t)-\frac{\beta_{i}}{\beta_{k} \mu\left(A_{k}\right)} f_{k}(t)\right)+\sum_{i \in S^{j}} f(t) \chi_{A_{i}} .
\end{aligned}
$$

Finally, note that $\left(\frac{1}{\mu\left(A_{i}\right)} f_{i}-\frac{\beta_{i}}{\beta_{k} \mu\left(A_{k}\right)} f_{k}, 0\right)$ belongs to $\mathcal{L}_{j}$, whatever $i \in S_{j}$, $i \neq k$ may be.

Second case. Suppose now that $y \neq 0$. Let $J_{j}=\left\{h \in \mathcal{H}_{j}^{\omega} \mid y_{h} \neq \emptyset\right\}$. If $J_{j}=\emptyset$, we are in the first case. So, consider $j$ such that $J_{j} \neq \emptyset$, and let $S(j)=S^{j} \cup S_{j}$. On the other hand, there exists $k \in S_{j}$, i.e., $\alpha_{k} \neq 0$. Consider $h_{k} \in H_{k}$. By definition of $\mathcal{L}$, one has $\alpha_{k}=-\frac{1}{\beta_{k}} \sum_{i \neq k} \alpha_{i} \beta_{i}+\frac{1}{\beta_{k}} \sum_{h \in \mathcal{K}_{j}^{\omega}} p_{h}^{*} y_{h}$. For each $h \in \mathcal{H}_{j}^{\omega}$ such that $h \neq h_{k}$, consider now $A_{i(h)} \subset \mathcal{I}_{j}^{\omega}$, such that $h \in H_{i(h)}$. So, if $t \in \mathcal{I}_{j}^{\omega}$, then $f(t)$ can be written as follows

$$
\begin{aligned}
\left(f \cdot \chi_{T_{j}^{\omega}}\right)(t)= & -\frac{1}{\beta_{k} \mu\left(A_{k}\right)}\left(\sum_{\substack{i \in S_{j} \\
i \neq k}} \alpha_{i} \beta_{i}\right) f_{k}(t)+\frac{1}{\beta_{k} \mu\left(A_{k}\right)}\left(\sum_{h \in \mathcal{H}_{j}^{\omega}} p_{h}^{*} y_{h}\right) f_{k}(t) \\
& +\sum_{\substack{i \in S_{j} \\
i \neq k}} \frac{\alpha_{i}}{\mu\left(A_{i}\right)} f_{i}(t)+\sum_{i \in S^{j}} f(t) \chi_{A_{i}} \\
= & \frac{p_{h_{k}}^{*} y_{h_{k}}}{\beta_{k} \mu\left(A_{k}\right)} f_{k}(t)+\sum_{\substack{i \in S_{j} \\
i \neq k}} \frac{\alpha_{i}}{\mu\left(A_{i}\right)} f_{i}(t)-\sum_{\substack{i \in S_{j} \\
i \neq k}} \frac{\alpha_{i} \beta_{i}}{\beta_{k} \mu\left(A_{k}\right)} f_{k}(t) \\
& +\sum_{\substack{h \in \mathcal{H}_{j}^{\omega} \\
h \neq h_{k}}} \frac{p_{h}^{*} y_{h}}{\beta_{k} \mu\left(A_{k}\right)} f_{k}(t)+\sum_{i \in S^{j}} f(t) \chi_{A_{i}}
\end{aligned}
$$




$$
\begin{aligned}
= & \frac{p_{h_{k}}^{*} y_{h_{k}}}{\beta_{k} \mu\left(A_{k}\right)} f_{k}(t)+\sum_{\substack{i \in S_{j} \\
i \neq k}} \alpha_{i}\left(\frac{1}{\mu\left(A_{i}\right)} f_{i}(t)-\frac{\beta_{i}}{\beta_{k} \mu\left(A_{k}\right)} f_{k}(t)\right) \\
& +\sum_{\substack{h \in \mathcal{H}_{j}^{\omega} \\
h \neq h_{k}}} \frac{p_{h}^{*} y_{h}}{\beta_{k}}\left(\frac{1}{\mu\left(A_{k}\right)} f_{k}(t)-\frac{\beta_{k}}{\beta_{i(h)} \mu\left(A_{i(h)}\right)} \chi_{A_{k}}\right) \\
& \sum_{\substack{h \in \mathcal{H}_{j}^{\omega} \\
h \neq h_{k}}} \frac{p_{h}^{*} y_{h}}{\beta_{i(h)} \mu\left(A_{i(h)}\right)} \chi_{A_{i(h)}}+\sum_{i \in S^{j}} f(t) \chi_{A_{i}} .
\end{aligned}
$$

So, for each connected component $j$, one has

$$
\begin{aligned}
\left(\left(f \cdot \chi_{T_{j}^{\omega}}\right)(t),\left(y_{h}\right)_{h \in \mathcal{H}_{j}^{\omega}}\right)= & \sum_{i \in S_{j}^{j}}\left(f_{i}(t), 0\right)+\frac{y_{h_{k}}}{\beta_{k}}\left(\frac{p_{h_{k}}^{*}}{\mu\left(A_{k}\right)} f_{k}(t), \beta_{k} e^{h_{k}}\right) \\
& +\sum_{\substack{i \in \mathcal{S}_{j} \\
i \neq k}} \alpha_{i}\left(\frac{1}{\mu\left(A_{i}\right)} f_{i}(t)-\frac{\beta_{i}}{\beta_{k} \mu\left(A_{k}\right)} f_{k}(t), 0\right) \\
& +\sum_{\substack{h \in \mathcal{H}^{\omega} \\
h \neq h_{k}}} \frac{p_{h}^{*} y_{h}}{\beta_{k}}\left(\frac{1}{\mu\left(A_{k}\right)} f_{k}(t)-\frac{\beta_{k}}{\beta_{i(h)} \mu\left(A_{i(h)}\right)} \chi_{\left.A_{k}, 0\right)}\right. \\
& +\sum_{\substack{h \in \mathcal{H}_{j}^{\omega} \\
h \neq h_{k}}} \frac{y_{h_{k}}}{\beta_{i(h)}}\left(\frac{p_{h}^{*}}{\mu\left(A_{i(h)}\right)} \chi_{\left.A_{i(h)}, \beta_{i(h)} e^{h}\right)}\right.
\end{aligned}
$$

Note that if $i \in S^{j}$, then $\left(f_{i}, 0\right) \in \mathcal{L}_{0}$. Note also that $\left(\frac{p_{h_{k}}^{*}}{\mu\left(A_{k}\right)} f_{k}(t), \beta_{k} e^{h_{k}}\right)$, $\left(\frac{p_{h}^{*}}{\mu\left(A_{i(h)}\right)} \chi_{A_{\mathrm{i}(h)}}, \beta_{i(h)} e^{h}\right)$ belong to $\mathcal{L}^{j} ;$ and $\left(\frac{1}{\mu\left(A_{i}\right)} f_{i}(t)-\frac{\beta_{i}}{\beta_{k} \mu\left(A_{k}\right)} f_{k}(t), 0\right)$, $\left(\frac{1}{\mu\left(A_{k}\right)} f_{k}(t)-\frac{\beta_{k}}{\beta_{i(h)} \mu\left(A_{i(h)}\right)} \chi_{A_{k}}, 0\right)$ belong to $\mathcal{L}_{j}$.

\section{References}

[1] Artstein, Z. (1979): “A note on Fatou's lemma in several dimensions", Journal of Mathematical Economics 6 (3), 277-282.

[2] Aumann,R.J. (1966): "Existence of competitive equilibria in markets with a continuum of traders", Econometrica 34 No 1, 1-17.

[3] Beauzamy, B. (1985): Introduction to Banach Spaces and their Geometry, North-Holland Mathematics Studies 68. 
[4] Bonnisseau, J. M. and Jofré, A (1994): "Equilibrium manifold for linear exchange economies", Cahier Eco-Math, Université de Paris 1.

[5] Cartan, H. (1977): Cours de Calcul Differentiel, Hermann, Paris.

[6] Champsaur, P. and B. Cornet (1990): "Walrasian exchange processes", in Economic Decision-Making : Games, Econometrics and Optimization, (Gabszewicz, Richard and Wolsey Eds.), Elsevier Science Publishers B.V.

[7] Cheng, H-C. (1979): "Linear economies are 'gross substitute' systems", Journal of Economic Theory 20, 110-117.

[8] Cornet, B. (1989): "Linear exchange economies", Cahier Eco-Math, Université de Paris 1.

[9] DiERKER, H. (1975): "Equilibria and core of large economies", Journal of Mathematical Economics 2, 155-169.

[10] Gale, D. (1957): "Price equilibrium for linear models of exchange", Technical Report P-1156, The Rand Corporation.

[11] Gale, D. (1960): The Theory of Linear Economic Models, Academic Press.

[12] Gale, D. (1976): "The linear exchange model", Journal of Mathematical Economics 3, 205-209.

[13] Eaves B.C. (1976): "A finite algorithm for the linear exchange model", Journal of Mathematical Economics 3, 197-203.

[14] Hildenbrand, W. (1974): Core and Equilibria of a Large Economy, Princeton University Press.

[15] KöthE G. (1969): Topological Vector Spaces I, Springer-Verlag, New York Inc.

[16] McKenziE, L. (1960): "Matrices with dominant diagonals and economic theory", in Mathematical Methods in the Social Sciences (Arrow, Karlin and Suppes, Eds), Stanford University Press.

[17] Mertens, J.M. (1995): "The limit-price mechanism" (Notes)

[18] NiKaIDo, H. (1968): Convex Structures and Economic Theory, Academic Press. 